\section{Professor D. G. Harnden}

Professor Harnden was Assistant Editor of the Journal from November 1971 to April 1979 and during this time he was responsible for all the papers on cytogenetics. This involved a very great deal of work, and I am sure readers would wish to join me in thanking him officially and publicly for his great services to the Journal.
Professor Harnden remains on the Editorial Committee and his place as Assistant Editor has been taken by Dr Martin Bobrow, whom we welcome.

\section{Professor Arnold Sorsby}

We congratulate Professor Sorsby, who was Editor of the Journal from 1964 to 1969, on his Honorary Doctorate in the City University.

C. A. CLARKE 